\title{
Development and control of a robotic arm for percutaneous surgery
}

\author{
Chunlin Zhou \\ College of Control Science and Engineering, Zhejiang University, Hangzhou, China \\ Huifeng Wu \\ College of Computer Science and Technology, Hangzhou Dianzi University, Hangzhou, China \\ Xiang Xu, Yong Liu and Qi Zhu \\ College of Control Science and Engineering, Zhejiang University, Hangzhou, China, and \\ Shuwen Pan \\ Zhejiang University City College, Hangzhou, China
}

\begin{abstract}
Purpose - The purpose of this paper is to propose a robotic system for percutaneous surgery. The key component in the system, a robotic arm that can manipulate a puncture needle is presented. The mechanical design, the motion control and the force control method of the robotic arm are discussed in the paper.

Design/methodology/approach - The arm with an arc mechanism placed on a 3D Cartesian stage is developed as a puncture needle manipulator to locate the position of the needle tip, tune the needle's posture and actuate the puncture motion under the visual guidance of two orthogonal X-ray images of a patient by a surgeon. A focusing method by using two laser spots is proposed to automatically move the needle tip to a surgery entry point on the skin. A dynamics model is developed to control the position of the needle mechanism and an explicit force control strategy is utilized to perform the needle insertion.

Findings - With the surgical system, a surgeon can easily perform puncture operation by taking two orthogonal real-time X-ray images as a visual feedback and accurately navigating the needle insertion. The laser-guided focusing method is efficient in placement of the needle tip. The explicit force control strategy is proved to be effective for holding constant and stable puncture force in experiments.

Originality/value - The robotic arm has an advantage in easy redirection of the needle because the rotation and the translation are decoupled in the mechanism. By adopting simple laser pens and a well-developed kinematics model, the system can handle the entry point, locating task automatically. The focusing method and the force control method proposed in the paper are useful for the present system and could be intuitive for similar surgical robots.
\end{abstract}

Keywords Surgical robot, Percutaneous surgery, X-ray

Paper type Research paper

\section{Introduction}

As a normal sector in most minimally invasive surgeries (MIS) (Adhami and Coste-Manière, 2003), percutaneous puncture is the important factor contributing to the effect of the treatment. It has gained rapid development since MIS was first brought forward in the 1980s (Wickham, 1987). With reduced trauma than traditional open surgery, MIS is associated with less pain, less scarring, quick recovery and less post-op complications. By extending surgeon's ability to manipulate surgical instruments more stably and accurately in surgery, MIS enjoys an unprecedented boost in operation quality (Taylor and Stoianovici, 2003; Moustris et al., 2011). Today, minimally invasive surgery operated by surgical robots is widely applied in

The current issue and full text archive of this journal is available on Emerald Insight at: www.emeraldinsight.com/0144-5154.htm

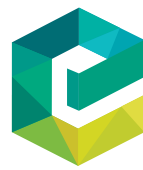

Assembly Automation 37/3 (2017) 314-321

Emerald Publishing Limited [ISSN 0144-5154]

[DOI 10.1108/AA-12-2016-179] diverse surgical specialties like orthopedics, neurosurgery, gynecology and urology (Mack, 2001).

In the puncture operation, the surgeon needs to guide an instrument about a single entry point with flexible orientation mobility. This kinematic manipulation, called remote center of motion (RCM), is suitable to be accomplished by a robot. Traditional serial manipulators (Kwoh et al., 1988; Yang et al.,

(C) Chunlin Zhou, Huifeng Wu, Xiang Xu, Yong Liu, Qi Zhu and Shuwen Pan. Published by Emerald Publishing Limited. This article is published under the Creative Commons Attribution (CC BY 4.0) licence. Anyone may reproduce, distribute, translate and create derivative works of this article (for both commercial \& non-commercial purposes), subject to full attribution to the original publication and authors. The full terms of this licence may be seen at http://creativecommons.org/licenses/by/4.0/ legalcode

The financial supports by National Natural Science Foundation of China (51305396 and U1509210), the Fundamental Research Funds for the Central Universities, and the Open Research Project of the State Key Laboratory of Industrial Control Technology, Zhejiang University, China are acknowledged.

Received 26 December 2016

Revised 17 March 2017

Accepted 24 March 2017 
2010) can finish the redirecting task by running matrix computations by solving inverse kinematics. A drawback of this approach is that all joints will be involved in motion during redirection because joints are generally serially linked, which causes complications in motion controls ( $\mathrm{Li}$ et al., 2010). Additionally, light motion of the end-effector may cause sharp joint transition in joint space which is introduced by the singularity. The complexity preventing such robots from clinic applications because serially connected robotic arm is not easily tele-controlled by a surgeon especially when the degrees of freedoms (DOFs) are multiple. Automatic operation may be a solution to tackle the problem since the inverse-kinematics of a multi-DOF robot can be computed by control software. Unfortunately, due to strict ethical standards and extremely high reliability requirements in surgical equipment, the automatic operation by a robot and its intelligent control system is not accepted in most cases. Therefore, ease of manipulation is a necessary criterion when developing a surgical robot.

For safety reasons, customized and remote-controlled RCM schemes are brought forward to avoid these potential hazards. Usually, RCM mechanisms have two to three (the spin DOF of the insertion needle is useless in certain conditions) rotation DOFs and a translational DOF for penetration (Li et al., 2010; Hannaford et al., 2013). Mitchell and Koo et al. (Mitchell et al., 2007) developed an RCM robot for retinal surgery with a long tubular arm attaching separated roll and tilt mechanisms. Kuo and Dai (Kuo and Dai, 2012) presented a parallel manipulator with a motion constrained leg and two auxiliary actuation legs. Virtual RCM implemented by software is also achieved in robot with magnetic tracker (Boctor et al., 2004) or through offline medical images such as MRI or CT scans (Monfaredi et al., 2014).

A robotic system for percutaneous surgery contains many different functional components among of which the guidance and manipulation devices are the most important parts. In this paper, we present a robotic arm with an arc mechanism placed on a 3D Cartesian stage as the puncture manipulator (Figure 1). The arc-arm is used to tune the posture and perform puncture motion. The stage is used to

Figure 1 Photo of an X-ray image guided robotic system for percutaneous surgery in a radiation-proofed room

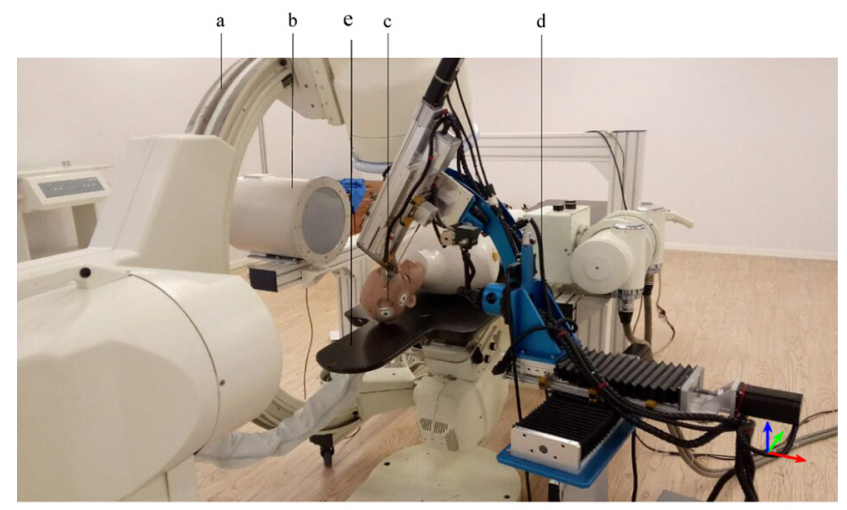

Notes: (a) An upper X-ray unit; (b) a lateral X-ray unit; (c) a manikin for brain puncture test; (d) a robotic arm; and (e) a medical bed locate the position of the needle point. An extra linear actuator is attached on the arc to perform the needle insertion. This design makes the translation motion and the rotation motion in the manipulation of the puncture needle decoupled. Two X-ray devices are orthogonally placed to provide real-time image guidance for a surgeon to track the needle tip and tune its posture under a visual surveillance. An automatic surgical bed is used to deliver the patient. Together with well-designed control hardware and software, an $\mathrm{X}$-ray image guided robotic system for percutaneous surgery can be built, as depicted by Figure 1 .

The upper X-ray unit [Figure 1(a)] provides a top view of the operation field, and the lateral unit [Figure 1(b)] provides a side view. Each image provides a $2 \mathrm{D}$ position information of the needle point, and two orthogonal images together provide totally $3 \mathrm{D}$ information (one $\mathrm{DOF}$ is redundant). The robotic arm [Figure $1(\mathrm{~d})$ ] is remotely controlled by a surgeon who can judge the tip position and postures of the needle by observing the two X-ray images, which greatly reduces the complex pre-planning of the robotic operation (Hayashibe et al., 2005). A test manikin [Figure 1(c)] lies on an automatic medical bed [Figure 1(e)]. Two views of X-ray devices can be modulated by control the motion of the bed.

With the equipment in Figure 1(a), successful puncture operation relies much on the control of the robotic arm (Berkelman et al., 2005). There are two major issues involved in the control. The first one is to make the needle tip coincident with the desired entry point on the skin surface with a right posture. The position of the entry point is usually determined by the surgeon. This process is called focusing. Manual focusing based on trial-and-error procedure is tedious and the accuracy cannot be guaranteed. In this paper, we propose an easy way to automatically fulfill this task by using a laser-guided method.

The second issue is the force control during the needle penetrating the skin. To reduce the risk that the needle hurts patients, elastic connection between the needle and its actuator is employed. This configuration increases the compliance of the puncture process and provides a safer operation than that using a rigid connection. However, it brings difficulties in contact force control, which is an important requirement in puncture operation. An explicit force control strategy (Volpe and Khosla, 1993) is employed to achieve the stable and precise contact force for the compliant mechanism.

The following sections describe the design of the robot, the motion control method and the puncture force control method. In Section II, design of the robotic arm will be introduced. System's kinematics and the auto-focusing method will be presented in Section III. The design of a force controller for puncture process will be discussed in Section IV. In Section V, experimental results will be presented. The paper will be summarized in Section VI.

\section{Mechanical design of the robotic arm}

From mechanical point of view, there are at least 6 DOFs required in a robotic arm for puncture operation, which is much similar to industrial robots. However, we do not recommend a 6-DOF industrial robot in percutaneous surgery. When performing insertion of needle, i.e. when the 
end-effector of an industrial robot performs straight line motion, all six joints are involved in the motion. This situation decreases the reliabilities because a tiny motion error of any joints may cause a failure of the surgery and then may hurt the patient. For a surgical robotic arm, we think, only one joint is allowed to move and all other ones should be firmly fixed when inserting a needle. Therefore, the surgical robotic arm will have three DOFs for 3D manipulation of needle tip, two DOFs for pose placement and one DOF for insertion of the needle. We propose a robot composed of four major components, as shown in Figure 2. The needle penetrates skin through an entry point on the skin surface with a particular posture. The robot is used to locate the tip point of the needle to coincide with the entry point and tune its penetration posture. Six DOFs are required: three translational motion for positioning of needle tip, two rotational motion for posing and one translation for the penetration.

The $\mathrm{Z}$-axis movement is implemented from reformation of an off-the-peg lifting table. Lead screws driven by servo motors produce the X-Y axes movement. Wheels are mounted below the platform which enhance the mobility of the system in the operating room. The distal rotary arm served for the redirection of the needle has a rotary base along axis-X. While an arc arm is extended from the base to enlarge the scope of operation during surgery, an arc sliding rail attached to the arm provides tilt degree of freedom of the needle drive unit. Needle drive unit is planned for powering the active motion of the percutaneous needle. A linear motor attached with a needle carrier is used for penetration movement. Each DOF has three proximity detectors assembled to limit its motion range and pass back joint's position information.

The robot can be operated manually or automatically. In the first manner, the robot is controlled by a surgeon through a tele-operation handler. The doctor will judge the right pose and needle tip position by visual observations and manipulated the handler to control the movement of the robot. In the second mode, automatic positioning of the needle is achieved by using two laser pointers, as depicted in Figure 2. The light beams of the two laser pointers are

Figure 2 Design of a robotic arm for percutaneous surgery

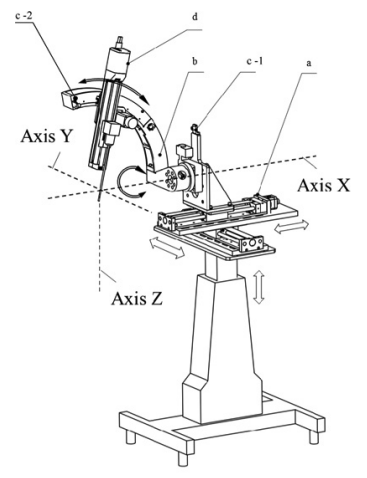

Notes: (a) A 3-D Cartesian motion stage; (b) a rotary arc arm; (c) guiding laser pointers; (d) a needle driving unit designed to intersect at the ball center of the rotary arm, i.e. the intersection point of axis- $\mathrm{X}$ and the path line of the needle.

It can be seen from Figure 2 that the puncture needle always aims at the spherical center point of the arc structure. This center point has also been set as the entry point on the skin surface. The configuration makes it possible to decouple rotary motions and the linear motions during movement of the robotic arm. This means a surgeon can first tune the position of the arc center to make it coincident with the entry point by controlling motions of the proximal Cartesian motion stage without causing any posture change. After that the surgeon can tune the two rotary DOFs to achieve desired puncture pose without causing a detachment of the entry point and the arc center. This property brings an easy manipulation for surgeons.

\section{Motion control method}

\subsection{Basic kinematics}

In terms of geometry, the arm model is equivalent to a $2 \mathrm{R}$ manipulator with a translational end-effector (Figure 3). Origin of upper arm is defined at the center on the top plane of the rotary base. Radius of the arc is denoted by $r . \varphi$ denotes the rotation angle of the rotary base. Angle $\alpha$ is fixed to limit the lower bound of the tilt motion which is denoted by $\theta$. Travel distance of the needle carrier is denoted by $d$. Forward kinematic equations of the end-effector (the tip of the needle) position $p_{t}\left(x_{t}, y_{t}, z_{t}\right)$ are expressed as below:

$$
\begin{gathered}
x_{t}=r-(r-d) \cdot \cos (\alpha+\theta) \\
y_{t}=(r-d) \cdot \sin (\alpha+\theta) \cdot \cos \varphi \\
\left.z_{t}=(r-d) \cdot \sin (\alpha+\theta) \cdot \sin \varphi\right)
\end{gathered}
$$

\subsection{Focusing}

Results of traditional localization approaches rely heavily on the accuracy of positioning. Two laser pointers in the system help to reduce the complexity of this task. Two laser pens are placed in a way that their light beams must intersect at the spherical center point of the arc arm (see c-1 and c-2 in Figure 2). Additionally, the axial lines of the insertion needle and two light beams must be in the same plane, as illustrated in Figure 3. An industrial camera is set on the top of patient to detect the relative positions of two laser spots and the entry point. By using the position information of these three points, the needle tip can be automatically moved to the entry point. The targeting plan is performed through three steps.

Figure 3 Sketch of the upper rotary arm

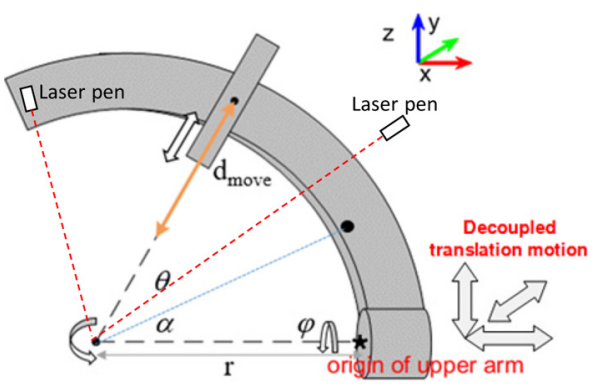


Step 1: In normal conditions, there might be two laser spots projected on the skin surface. As shown in Figure 4, ambiguity occurs when two spots come into the camera view. The intersection of two laser beams may be above or below the skin surface even if it looks similar in the 2D image acquired from the top camera. Nevertheless, the two cases in Figure 4 are interconvertible by movement along $\mathrm{Z}$. Without loss of generality, we take case 1 as the initial status. Top camera is placed horizontally and laser beams should also be tuned to lie on the sagittal plane (the $\mathrm{X}-\mathrm{Z}$ plane) before the formal manipulation starts.

Step 2: It is not convenient to move the intersection point of two laser beams directly to the entry point because the skin surface is curved and there is no depth information of the entry point through one top camera. Therefore, we manage to constrain the movement of laser beams toward entry point to a predictable and observable path in the camera's view. As we mentioned in step 1, the two laser beams (Lasers 1 and 2) as well as the intersection $(O)$ and their projection (LS1 and LS2) all lie on the sagittal plane intersecting with skin surface by a curve $S$, which is illustrated by Figure 5.

In this step, we need to move the connecting line of LS1 and LS2 to the entry point. In the X-Y plane, like what we see from the camera, two laser spots determine a unique straight-line parallel to axis $\mathrm{X}$ with the linear function representation $y=b$. The movement direction of the connecting line is defined by

Figure 4 Two cases when two laser spots projects on the skin
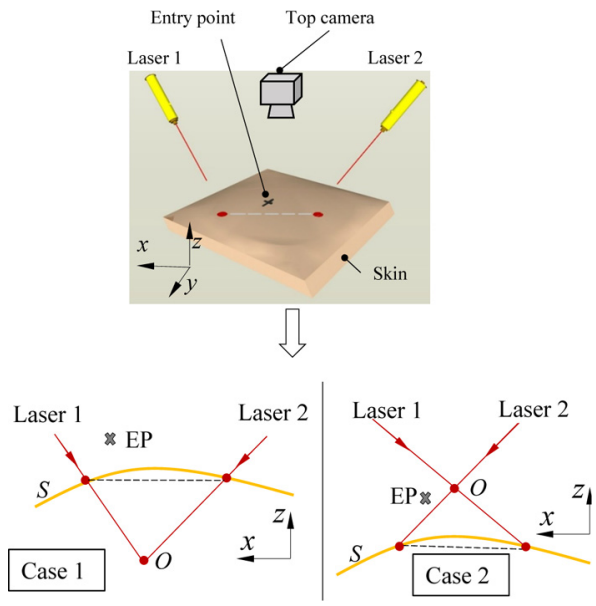

Figure 5 Moving scheme in X-Y plane

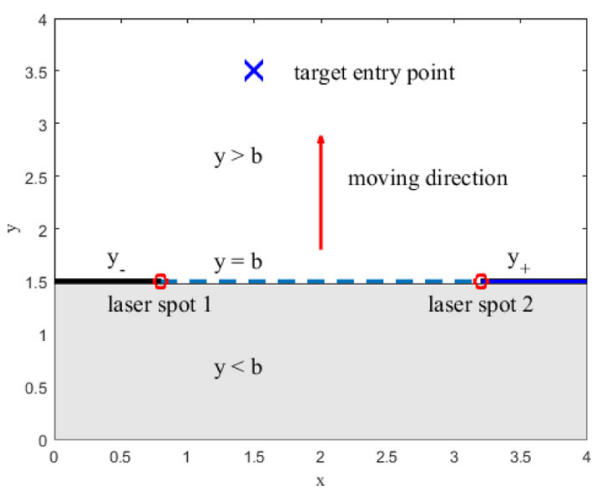

the line's position with respect to the entry point (Figure 5). The unit component velocity along $x$ and $y$ is given from the following formula.

Case 1: The target is outside the connecting line (Movement along the normal vector.):

$$
\begin{aligned}
& {\left[\begin{array}{ll}
d x & d y
\end{array}\right]^{T}=\left[\begin{array}{ll}
0 & d i r
\end{array}\right]^{T}} \\
& \text { dir }= \begin{cases}1 & y_{t g}>b \\
-1 & y_{t g}<b\end{cases}
\end{aligned}
$$

where the position of the entry point is given by $\left(x_{t g}, y_{t g}\right), b$ is the intercept of the line function.

Case 2: The target is on the connecting line but not located on the line segment (Movement along the tangent vector.):

$$
\begin{aligned}
& {\left[\begin{array}{ll}
d x & d y
\end{array}\right]^{T}=\left[\begin{array}{ll}
\operatorname{dir} & 0
\end{array}\right]^{T}} \\
& \operatorname{dir}= \begin{cases}-1 & x_{t g}<x_{L S 1} \\
1 & x_{t g}>x_{L S 2}\end{cases}
\end{aligned}
$$

Step 3: In this step, laser beams are moved toward entry point. Since the height of two laser points are unknown, the direction vector from intersection to entry point cannot be calculated. Instead, the direction of two laser beams are calculable when the pose of rotary arm is fixed (Figure 6). Movement along the direction of any laser beam reflects approaching of the other laser spot toward the entry point in the top view. The line will finally converge to the entry point in two steps by moving along the laser beams. The unit axial velocity for simultaneous motion along two laser beams are given as follows:

Figure 6 Moving steps for the focusing point to coincide with the entry point

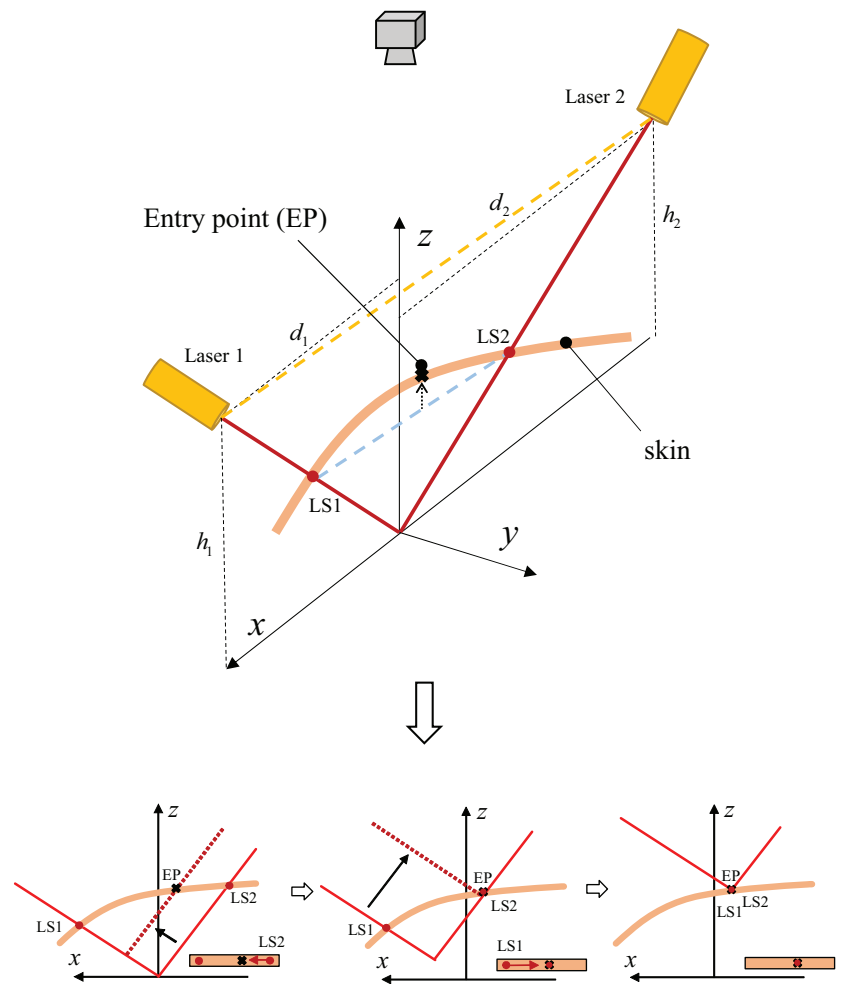




$$
\begin{aligned}
& {\left[\begin{array}{lll}
d x & d y & d z
\end{array}\right]^{T}=\left[\begin{array}{lll}
-d_{2} & 0 & h_{2}
\end{array}\right]^{T}} \\
& {\left[\begin{array}{lll}
d x & d y & d z
\end{array}\right]^{T}=\left[\begin{array}{lll}
d_{1} & 0 & h_{1}
\end{array}\right]^{T} .}
\end{aligned}
$$

In equation (6) and Figure $6, h_{i}$ and $d_{i}(i=1$ or 2$)$ denote the distance from the $i^{\text {th }}$ laser source to $\mathrm{X}$ and $\mathrm{Z}$.

\section{Design of a force controller}

Safety is the first criteria for a percutaneous surgery. Many safety considerations should be made during the design of the surgical robot. In manual puncture operation, a surgeon holds a needle by hands and can adjust the contact force, speed of insertion and the posture of the needle according to the reaction of the patient. When performing a robotic puncture, lack of force feedback may cause unexpected damage to the patient. To avoid the risk, the needle in the surgical robot is attached to its actuator through a compliant mechanism which brings safer operation because the compliance of the mechanism can provide sufficient time for the mechatronic devices to respond correctly when emergency occurs. A force sensor is equipped with the compliant connection to provide force feedback during insertion. The force control in the compliant needle insertion mechanism is also a necessity in the robotic surgery, which will be discussed in the rest part of the section.

\subsection{Dynamic model of the needle-actuator system}

The needle is driven by a linear actuator. A one-dimensional pressing force sensor is mounted on the movable base of the actuator and connects the needle. There is an elastic connection between the needle and a movable actuator base. The rigid needle, its actuator, the damped elastic connection between them can be regarded as a small system. This compliant mechatronics system can be described by a base-excitation model as shown in Figure 7.

The model describes a two-mass with spring and damping system. The compliance of the system is reflected by the spring stiffness $K . \Phi(t)$ denotes the power force provided by the actuator. The compliance between the needle and stage, reflected by the spring stiffness $K$, causes the asynchronous movement of the two rigid bodies, which are denoted by $z_{s}(t)$ and $z_{n}(t)$, respectively. The relative displacement of the two rigid bodies, $\Delta z(t)$, can be given by:

$$
\Delta z(t)=z_{s}(t)-z_{n}(t)
$$

Figure $7 \mathrm{~A}$ base-excitation model of the needle-stage system

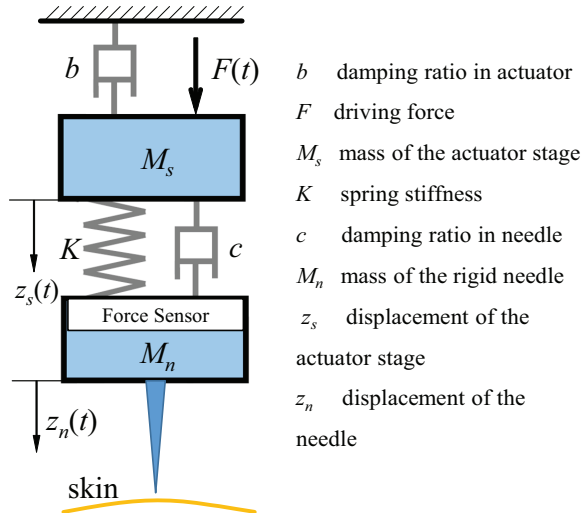

According to Newton's law, the following can be obtained:

$$
M_{n} \Delta \ddot{z}+c \Delta \dot{z}+K \Delta z=M_{n} \ddot{z}_{s}
$$

Combination of above two equations yields:

$$
M_{n} \ddot{z}_{n}+c \dot{z}_{n}+K z_{n}=-c \dot{z}_{s}-K z_{s}
$$

Note that in equation (9) the gravity force of the needle is absent because it can be compensated by the controller so as to simplify the derivation. By using Laplace transform, the transfer function of the open-loop position control plant can be written as:

$$
\frac{z_{n}(s)}{z_{s}(s)}=\frac{-c s-K}{M_{n} s^{2}+c s+K}
$$

The position control of the actuator's stage, $z_{s}(t)$, can be easily performed with a PID controller by assuming that it has a typical second-order model. The needle is serially linked with the stage, and equation (10) defines the dynamics of this link. With this equation, high-performance position control of the needle can also be obtained by using well-developed motion controllers.

\subsection{Design of a force controller}

When the needle tip contacts with the skin, the position control mode should promptly be switched into a force control mode. According to the base-excitation model, when contact occurs, the system dynamics can be given by:

$$
M_{s} \ddot{z}_{s}=-b \Delta \dot{z}-c \Delta \dot{z}-K \Delta z+F(t)
$$

A strategy called explicit force control (Volpe and Khosla, 1993) is adopted to perform the stable force tracking during the insertion. In this case, the force controller can be given by:

$$
F(t)=K_{p}\left(f_{d}-f_{s}\right)-K_{v} \Delta \dot{z}
$$

where $f_{d}$ is the desired force input, $f_{s}$ is the feedback of a force sensor, $K_{p}$ is a proportional coefficient and $K_{v}$ is a velocity coefficient.

The force controller is constructed by the contact force feedback, desired force input and a system state $\Delta z(t)$, which can be described by Figure 8 . By combining equations (11) and (12), we have:

$$
M_{s} \Delta \ddot{z}+M_{s} \Delta \ddot{z}_{n}+\left(b+c+K_{v}\right) \Delta \dot{z}+K \Delta z=K\left(f_{d}-f_{s}\right)
$$

During insertion, the movement speed of the puncture needle is kept very slow. In this situation, it can be assumed that the contact force has a simple expression, $f(t)=K \cdot \Delta z(t)$, and the acceleration term of the needle is approximately zero. Substituting these two conditions into equation (13) yields:

Figure 8 The explicit force control strategy

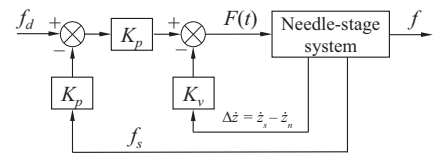




$$
M_{s} \ddot{f}+\left(b+c+K_{v}\right) \dot{f}+K f=K\left(K_{p} f_{d}-K f_{s}\right)
$$

Equation (14) implies that the velocity term $K_{v}$ tunes the equivalent damping of the system and thus affects the dynamical response. It can be proved that the steady state error $e_{s s}$ of the force control system is determine by the proportional coefficient, $K_{p}$, in means of:

$$
e_{s s}=\frac{f_{d}}{1+K_{p}}
$$

A lager $K_{p}$ leads to smaller steady state error. However, for an actual system, larger $K_{p}$ will also result in a saturation of the actuator. This problem can be fixed by using feedforward strategy, as illustrated by Figure 9. In this case, the force controller will be modified to equation (16):

$$
F(t)=K_{p}\left(f_{d}-f_{s}\right)-K_{v} \Delta \dot{z}+f_{d}
$$

\section{Experimental results}

The experimental devices include the robotic arm, an automatic bed, two orthogonally placed X-ray machines and a manikin instead of a human patient. The whole system is placed in a radiation-proof room (Figure 1). An operator (a surgeon) stays outside of the room to control the robotic arm by using a 6-DOF operating handle. By simultaneously observing two real-time X-ray images, the operator can accurately judge the actual position of the puncture needle, as illustrated by Figure 10. There are many aspects and a lot of operation procedures involved in a real percutaneous surgery. In this section, we focus on two experiments which test the auto-focusing method and the force tracking performance proposed in the paper. The experimental results are presented in the following parts of the section.

\subsection{Test of auto-focusing}

A simple experiment is designed to validate feasibility of the auto-focusing method by using laser pointers in supervision

Figure 9 Modified explicit force control strategy with a feedforward component

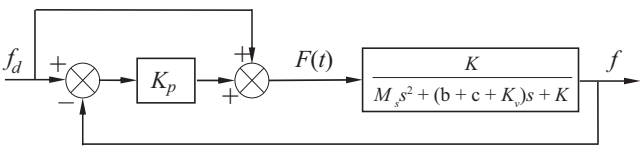

Figure 10 X-ray images in top view (left) and side view (right) of the manikin's head during needle insertion

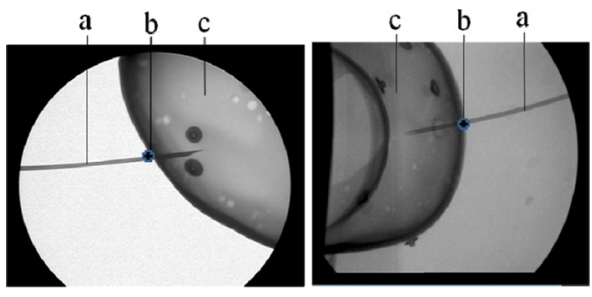

Notes: (a) The needle; (b) the entry point on the skin surface; (c) the head of the manikin for the test

mode (automatic mode). A spinal puncture needle is mounted on the needle driving unit. The needle is made up of 316 stainless steel and has a length of $80 \mathrm{~mm}$ and a diameter of $3.48 \mathrm{~mm}$. The positioning area is restricted to a $30 \mathrm{~mm} \times 20$ $\mathrm{mm}$ phenolic resin plate covered with a $1.5 \mathrm{~mm}$ thick silica gel. A 5 million-pixel CMOS industrial camera is mounted $25 \mathrm{~mm}$ high above the plate on a lifting support to obtain a comprehensive view of the positioning area.

In targeting stage, $\mathrm{X}-\mathrm{Y}-\mathrm{Z}$ translation motions are allowed to adjust the relative position of laser spots and entry point mark. Once the targeting phase is finished, the needle is driven slightly into the silica gel plate by the operator to leave a shallow mark for post-test measurement. In Figure 11(a) series of consecutive motions in the automatic positioning plan are presented. First, the joint line between two laser spots is moved to coincide with the interested entry point marked by " $X$ " in Figure 11(a). Then by using the control policy given by equation (5) the right laser spot in Figure 11(b) and (c) is moved to the entry point (EP). In Figure $11(\mathrm{~d})$ and (e), the left laser point moves to EP and in $11 \mathrm{f}$ two laser points and EP are finally coincident. After this process, the needle will accurately aim at the entry point. The average time dropped visibly to $8.46 \mathrm{~s}$ and average placement error is less than 0.8 $\mathrm{mm}$. The image process and the motor control software are implemented by using a Labview programing IDE and a Singleboard-RIO embedded controller.

\subsection{Test of the force tracking}

It is interesting that the force control is the more important than the position control in the robotic puncture. The puncture will terminate when the needle tip reaches the lesion area. Because of the safety concerns and the ethical issues, position control must be done by a surgeon. A surgeon will watch the real-time vision feedback to determine whether the needle tip reaches the lesion. The position control model given by equations (10) is only valid before the needle pierces the

Figure 11 Steps of automatic focusing by using two laser pointers in an experiment

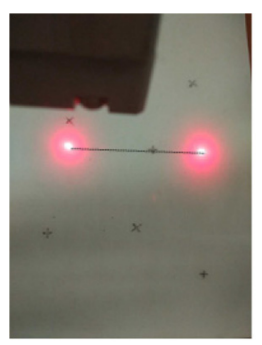

(a)

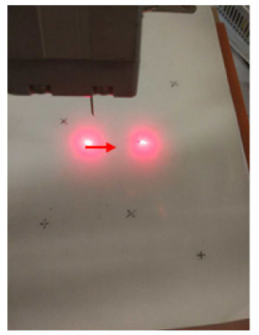

(d)

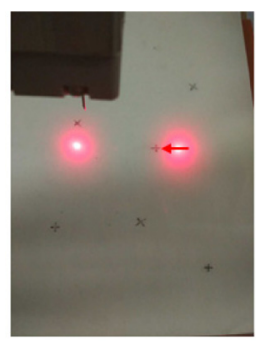

(b)

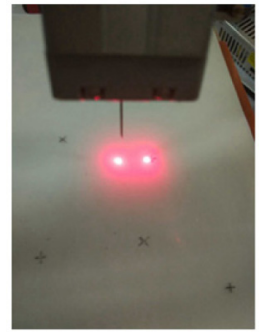

(e)

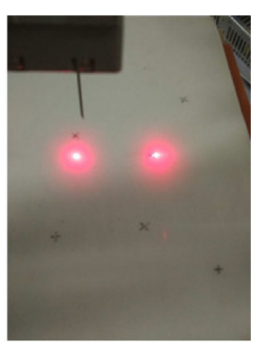

(c)

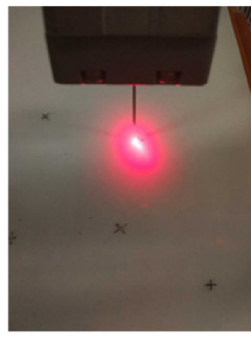

(f) 
skin. A common PID controller will be sufficient to perform the position control.

However, high-performance force control can hardly be achieved manually and has to be performed by well-designed control algorithm. For a percutaneous surgery, unstable insertion force is likely to damage the tissue where the needle passes. Additionally, due to the softness of muscles and skin, the position of a lesion (such as a liver tumor) hiding deeply beneath the skin will move to an unpredictable position when the needle presses the muscle. Accurate puncture requires an accurate estimation of the lesion's location. If the insertion force can be maintained stable, the position deviation of the lesion's position is also a constant and can be accurately calculated and compensated. Therefore, high-performance insertion force control is a necessity for a robotic puncture.

An experiment of puncture force control was conducted on the manikin's head. In such a puncture process, the piercing force must be larger than $300 \mathrm{~g}$ (determined by the material of the manikin). The puncture force should be maintained as approximately $600 \mathrm{~g}$ during the needle passing through the fiber tissues inside the manikin. This force mimics a real situation that an outer diameter $3.48 \mathrm{~mm}$ needle inserts into real human muscle tissues.

The robotic insertion is performed in an intermittent mode. There are two reasons for this action. The first one is that the surgeon needs to carefully watch the position of the needle and try to keep insertion deeper and deeper in a step-by-step manner, which ensures the safety of the operation. The second reason is that the pressure from the needle to the muscle tissue should be intermittently released to reduce the movement range of the lesion. Based on these facts, the experiment was designed in the way that the needle inserts the manikin by every $2 \mathrm{~s}$ after the needle pierced the skin. In the $1 \mathrm{st}$ second, a $600 \mathrm{~g}$ force command was applied to drive the insertion. In the 2 nd second, the force was released to let the pressed tissue get recovered.

Figure 12 shows an experimental result of the force tracking control, which causes a $67.8 \mathrm{~mm}$ insertion depth into the manikin's head in $10 \mathrm{~s}$. The overshoot of the step response is less than 7.65 per cent, setting time is less than $17.6 \mathrm{~ms}$. The

Figure $12 \mathrm{~A}$ record of insertion force tracking in $10 \mathrm{~s}$ under the controller given by equation (16)

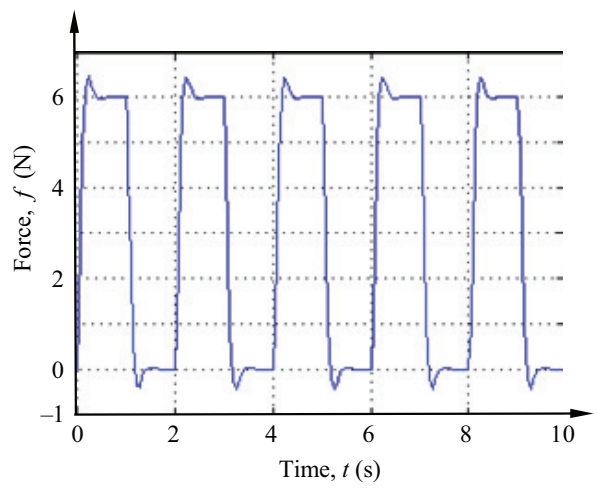

Notes: Parameters are set as $f_{d}=0.6 \mathrm{~N}, K_{p}=5$, $K_{v}=1,100, K=52,100, c=1.45 \mathrm{Ns} / \mathrm{m}$, $M=2.66 \mathrm{~kg}, b=61 \mathrm{Ns} / \mathrm{m}$ controller ensures a stable and constant force tracking to a series of $600 \mathrm{~g}$ intermittent force commands. It can be seen from Figure 12 that in the second half a force applying cycle, the system shows non-minimum-phase phenomenon. This can be explained by the compliance between the needle and its driving base. Such performance can be acceptable for the puncture operation.

\section{Conclusions}

Most of the surgery is too difficult to be accomplished yet by a robot even if the robotics communities have made remarkable progress in the past decades. The percutaneous puncture is relatively simple and it is easy for a robot to perform. However, this is not the reason why a robot is adopted. There are several clinical needs to push development of the robotic arm in this paper. In some surgery, such as the microwave ablation of a lung cancer, it is a critical challenge for a surgeon to insert the needle accurately to the target because there is no real-time guidance. The quality of the surgery completely depends on the surgeon's skills and experiences. The help of CT scanner or other X-ray devices may by useful to track the lung tumor which is moving along with the breathing. The problem is that the radiation is harmful for the surgeon who may often conduct similar surgeries. An effective solution is to use a tele-operated robotic system with guidance of real-time X-ray images, which is a contribution of the present paper. Another clinical need is that the manipulation of a surgical robot is must as easy as possible for a surgeon. Normally robotic arms-based revolute joints similar to industrial robots are adopted in medical application. These designs suffer from the complexity of manipulation. The robotic arm presented in this paper aims to solve this problem. The mechanism of the robot ensures the translation and the rotation of the arm decoupled, which offers easy manipulation. This is another contribution of the paper.

In this article, we proposed a design of stereotaxic surgery robot for percutaneous puncture. Compared with serial manipulator, redirection is much easier to be satisfied by an arc mechanism because the rotation motion and the translation motion are decoupled. The system can handle the entry point locating problem automatically, and complicated positioning system can be saved by adopting laser pens properly on the robot arm and a well-developed control policy. The navigation of the needle insertion can be achieved by using two orthogonal X-ray images in the teleoperation of the system. The system is considered quite intuitive and easy to be used since several auxiliary features are added to enhance its man-machine interaction capability when lacking of enough haptic and visual information. Force feedback and force control is merged into the master-slave manipulation device to help restore present touch sense of the surgeon. Experimental results show that the control policies discussed in the paper are effective and efficient.

The needle may bend during the puncture process. The focusing on the EP may not be accurate because the involuntary motion of a patient can move the EP. It is a challenge to address these practical problems and it will be done in further studies. From an engineering point of view, it worth designing a robotic system that can automatically finish a percutaneous surgery with no or little aids of professional 
surgeons. This requires research work in several aspects: real-time processing algorithms that helps to identify a lesion from CT or other X-ray images, an avigation system for the motion planning of robotic arms (including the coordinate movement of the hospital bed), compliant mechanisms of the puncture needle and multi-dimension position/force mixed control policies. These could be valuable for research in further studies. Future work will also focus on optimization of the whole system and clinical applications.

\section{References}

Adhami, L. and Coste-Manière, È. (2003), “Optimal planning for minimally invasive surgical robots", IEEE Transactions on Robotics and Automation, Vol. 19, pp. 854-863.

Berkelman, P., Boidard, E. and Cinquin, P., Okamura, A.M. and Fichtinger, G. (2005), "Control and user interface design for compact manipulators in minimally-invasive surgery", Proceedings of 2005 IEEE Conference on Control Applications Toronto, 28-31 August, IEEE, pp. 25-30.

Boctor, E.M., Webster, R.J., III., Mathieu, H., Okamura, A.M. and Fichtinger, G. (2004), "Virtual remote center of motion control for needle placement robots", Computer Aided Surgery, Vol. 9, pp. 175-183.

Hannaford, B., Rosen, J., Friedman, D.W., King, H., Roan, P., Cheng, L., Glozman, D., Ma, J., Kosari, S.N. and White, L. (2013), "Raven-II: an open platform for surgical robotics research", IEEE Transactions on Biomedical Engineering, Vol. 60, pp. 954-959.

Hayashibe, M., Suzuki, N., Hashizume, M., Kakeji, Y., Konishi, K., Suzuki, S. and Hattori, A. (2005), "Preoperative planning system for surgical robotics setup with kinematics and haptics", The International fournal of Medical Robotics and Computer Assisted Surgery, Vol. 1, pp. 76-85.

Kuo, C. and Dai, J.S. (2012), "Kinematics of a fully-decoupled remote center-of-motion parallel manipulator for minimally invasive surgery", fournal of Medical Devices, Vol. 6 No. 2, 21008.

Kwoh, Y.S., Hou, J. and Jonckheere, E.A. and Hayati, S. (1988), "A robot with improved absolute positioning accuracy for CT guided stereotactic brain surgery", IEEE Transactions on Biomedical Engineering, Vol. 35, pp. 153-160.
Li, G., Wu, D., Ma, R., Huang, K. and Du, Z. (2010), "Pose planning for robotically assisted minimally invasive surgery", 2010 3rd International Conference on Biomedical Engineering and Informatics, Yantai, 16-18 October, pp. 1769-1774.

Mack, M.J. (2001), "Minimally invasive and robotic surgery", fAMA, Vol. 285, pp. 568-572.

Mitchell, B., Koo, J., Iordachita, I., Kazanzides, P., Kapoor, A., Handa, J., Hager, G. and Taylor, R. (2007), "Development and application of a new steady-hand manipulator for retinal surgery", Proceedings 2007 IEEE International Conference on Robotics and Automation, Roma, 10-14 April, pp. 623-629.

Monfaredi, R., Seifabadi, R., Iordachita, I., Sze, R., Safdar, N.M., Sharma, K., Fricke, S., Krieger, A. and Cleary, K. (2014), "A prototype body-mounted MRI-compatible robot for needle guidance in shoulder arthrography", 5th IEEE RAS/EMBS International Conference on Biomedical Robotics and Biomechatronics, Sao Paulo, 12-15 August, pp. 40-45.

Moustris, G.P., Hiridis, S.C. and Deliparaschos, K.M. and Konstantinidis, K.M. (2011), "Evolution of autonomous and semi-autonomous robotic surgical systems: a review of the literature", The International fournal of Medical Robotics and Computer Assisted Surgery, Vol. 7, pp. 375-392.

Taylor, R.H. and Stoianovici, D. (2003), "Medical robotics in computer-integrated surgery", IEEE Transactions on Robotics and Automation, Vol. 19, pp. 765-781.

Volpe, R. and Khosla, P. (1993), "A theoretical and experimental investigation of explicit force control strategies for manipulators", IEEE Transactions on Automatic Control, Vol. 38, pp. 1634-1650.

Wickham, J.E. (1987), "The new surgery", British Medical fournal, Vol. 295 No. 6613, pp. 1581-1582.

Yang, L., Chng, C.B., Chui, C.K. and Lau, D.P.C. (2010), "Model-based design analysis for programmable remote center of motion in minimally invasive surgery", 2010 IEEE Conference on Robotics, Automation and Mechatronics, Singapore, 28-30 fune, pp. 84-89.

\section{Corresponding author}

Huifeng Wu can be contacted at: whf@hdu.edu.cn

For instructions on how to order reprints of this article, please visit our website: www.emeraldgrouppublishing.com/licensing/reprints.htm Or contact us for further details: permissions@emeraldinsight.com 